\title{
Schwinger-Dyson Equations and Dynamical gluon mass generation
}

\author{
A. C. Aguilar* and A. A. Natale ${ }^{\dagger}$ \\ ${ }^{*}$ Departamento de Física Teórica, Universidad de Valencia-CSIC \\ E-46100, Burjassot, Valencia, Spain \\ ${ }^{\dagger}$ Instituto de Física Teórica, Universidade Estadual Paulista \\ Rua Pamplona 145, 01405-900, São Paulo, SP, Brazil
}

\begin{abstract}
.
We discuss the solutions obtained for the gluon propagador in Landau gauge within two distinct approximations for the Schwinger-Dyson equations (SDE). The first, named Mandelstam's approximation, consist in neglecting all contributions that come from fermions and ghosts fields while in the second, the ghosts fields are taken into account leading to a coupled system of integral equations. In both cases we show that a dynamical mass for the gluon propagator can arise as a solution.
\end{abstract}

Keywords: Nonperturbative QCD; Gluon Schwinger-Dyson Equation; Infrared Gluon Propagator PACS: 12.38 -t, 11.15.Tk

The Schwinger-Dyson equations (SDE) are compound by an infinite set of the coupled equations that embody the full structure of the theory and provide in the continuum the appropriate framework to describe the behavior of the Green functions which are beyond of the scope of perturbative theory. In particular, the SDE can shed some light on the infrared (IR) properties of Quantum Chromodynamics (QCD) which can only be accessed through non-perturbative methods.

The structure of this tower of equations is such that it relates the n-point Green function to the $(\mathrm{n}+1)$-point function which naturally must satisfy, in its turn, its own SDE and so on. Since we were not able to deal with this infinite system of equations, some approximations must be imposed in order to obtain a tractable system.

Many attempts have been made to understand the IR gluon propagator behavior through the SDE. In the late seventies Mandelstam initiated the study of the gluon SDE in Landau gauge [1]. Neglecting the ghost fields contribution and imposing cancellations of certain terms in the gluon polarization tensor, he found a highly singular gluon propagator in the infrared. However, these results are discarded by simulations of QCD on the lattice at $95 \%$ confidence level [2], where it is shown that the gluon propagator is probably infrared finite.

Infrared finite solutions are also found in the Schwinger-Dyson approach, as result of different procedures. A gluon propagator endowed with a dynamical mass was obtained by Cornwall utilizing the "pinch technique" [3] while solving the gluon-ghost coupled system an infrared vanishing gluon propagator behavior was found by the authors of Ref. [4].

Here we show that in the Mandelstam and in the gluon-ghost coupled system approximations a dynamical gluon mass arise as another possible solution if we adopt a three gluon vertex with a massless pole and follow a specific renormalization procedure [See 


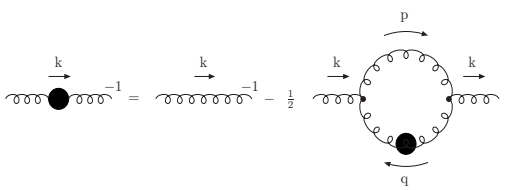

FIGURE 1. The Schwinger-Dyson equation in Mandelstam's approximation.

The full gluon and ghost propagators in Landau gauge are given by

$$
D^{\mu v}\left(q^{2}\right)=\left(\delta^{\mu v}-\frac{q^{\mu} q^{v}}{q^{2}}\right) \frac{\mathscr{Z}\left(q^{2}\right)}{q^{2}} \quad \text { and } \quad D_{G}(k)=-\frac{\mathscr{F}\left(k^{2}\right)}{k^{2}} .
$$

where $\mathscr{Z}\left(k^{2}\right)$ and $\mathscr{F}\left(k^{2}\right)$ are the dressing of the gluon and ghost propagators respectively. When we set $\mathscr{Z}\left(k^{2}\right)=\mathscr{F}\left(k^{2}\right)=1$ we recover the perturbative expressions for the propagators at tree level. Therefore these functions measure the transition from the nonperturbative to the perturbative regimes as their values change with the scale.

The truncation scheme known as Mandelstam approximation consists in neglecting the ghosts fields and all terms with four-gluon interactions, and is pictorially represented in Fig.(1). The infinite contribution to the gluon SDE in this approximation is shown in the left-hand side of Eq.(2) together with the gluon propagator renormalization constant $Z_{3}$. On the right-hand side we show the behavior of the finite contribution $\left(1+\frac{\kappa}{k^{2}}\right)$ after the infinite contribution is subtracted with the help of the constant $Z_{3}$

$$
Z_{3}-\frac{\alpha\left(\mu^{2}\right)}{4 \pi} \int_{k^{2}}^{\Lambda^{2}} d q^{2} 7 q^{2} D\left(q^{2}\right)=1+\frac{\kappa}{k^{2}} .
$$

The details of this procedure are explained in the Ref.[5], and it allows for massive solutions of the SDE. We obtain the curves for the gluon propagator, $D\left(q^{2}\right)$, as a function of the momentum $q^{2}$ which are plotted in the left panel of Fig.(2). Theses curves were produced utilizing different values to the renormalization point $\mu^{2}$. The external curves delimit the lower and the higher values of the $\Lambda_{Q C D}$ in the range $[182,557] \mathrm{MeV}$ which is fixed by the choice of $\mu^{2}$. These numerical solutions for the gluon propagator can be fitted by the following equations

$$
D\left(q^{2}\right)=\frac{1}{q^{2}+\mathscr{M}^{2}\left(q^{2}\right)}, \quad \text { where } \quad \mathscr{M}^{2}\left(q^{2}\right)=\frac{m_{0}^{4}}{q^{2}+m_{0}^{2}} .
$$

where $\mathscr{M}^{2}\left(q^{2}\right)$ is the gluon dynamical mass.

Analising the ratio $m_{0} / \Lambda_{Q C D}$ which give to us a better idea about dependency on the renormalization point of our solution, we have found an average value $m_{0} / \Lambda_{Q C D} \approx 3$ that is consistent with the previous estimates for this mass [3].

On the other hand, if we take into account the ghost fields, following the same steps of Ref.[4] and applying the renormalization procedure explained in Ref.[5], we obtain, for the gluon fields, the same qualitative behavior shown in the left panel of Fig.(2), however the average value for the ratio $m_{0} / \Lambda_{Q C D}$ decreases to 2.5 . 

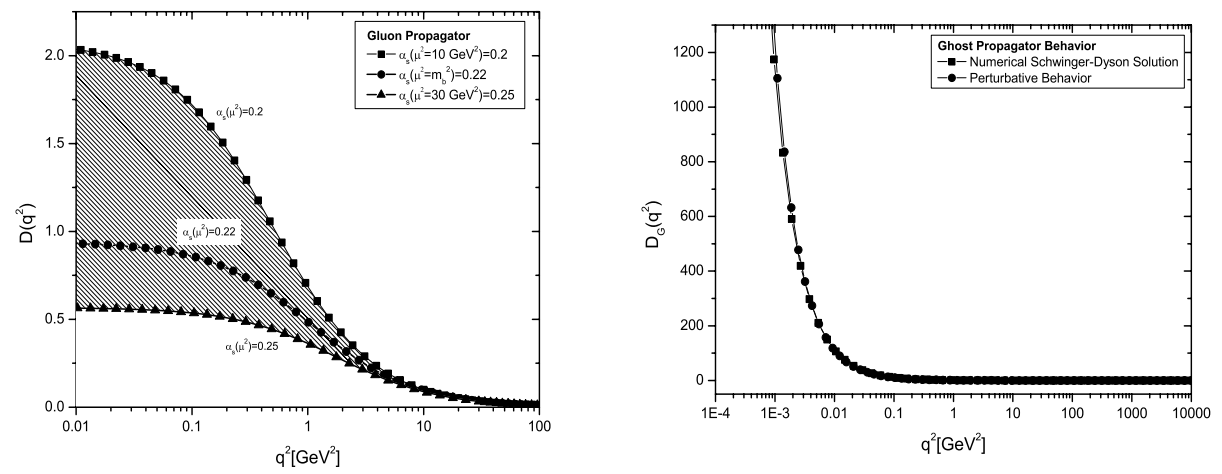

FIGURE 2. Left panel: Gluon propagator, $D\left(q^{2}\right)$, as function of momentum $q^{2}$ for different scales. The central curve (line + circle) was obtained when we fix the renormalization point, $\mu^{2}$, at bottom quark mass, $m_{b}^{2}=(4.5)^{2} \mathrm{GeV}^{2}$ with the central value of $\alpha\left(m_{b}^{2}\right)=0.22$. Right panel: The behavior of the ghost propagator, $D_{G}\left(q^{2}\right)$, obtained through the numerical solution of the Schwinger-Dyson equation, when $\alpha_{s}\left(\mu^{2}\right)=0.22$ at $\mu^{2}=\left(m_{\mathrm{b}}\right)^{2}=(4.5)^{2} \mathrm{GeV}^{2}$, together with its ultraviolet behavior.

Within this approximation, we can see that the ghost propagator develops practically the same perturbative behavior for all spectrum of momenta, as is shown in the right panel of Fig.(2).

\section{CONCLUSIONS}

We computed the SDE for the gluon propagator in the Landau gauge within two different approximations where the fermions and quartic vertices are neglected. The full triple gluon vertex is also extended to include the possibility of dynamical mass generation. We show, in both cases, that following a specific renormalization procedure, the gluon propagator develops a dynamical mass whose ratio with the $\Lambda_{Q C D}$ scale has an average value around two or three depending on the approximation utilized, such values are consistent with the previous estimates [3].

\section{ACKNOWLEDGMENTS}

This research was supported by the Conselho Nacional de Desenvolvimento Científico e Tecnológico (CNPq) (AAN), Fundação de Amparo à Pesquisa do Estado de São Paulo (FAPESP) and Coordenação de Aperfeiçoamento de Pessoal de Nível Superior (CAPES) (ACA).

\section{REFERENCES}

1. S. Mandelstam, Phys. Rev. D20 (1979) 3223.

2. P. Marenzoni, G. Martinelli, N. Stella, e M. Testa, Phys. Lett. B318 (1993) 511; C. Alexandrou, Ph. de Forcrand and E. Follana, Phys. Rev. D65 (2002) 114508; D65 (2002) 117502.

3. J. M. Cornwall, Phys. Rev. D26 (1982) 1453.

4. R. Alkofer and L. von Smekal, Phys. Rept. 353 (2001) 281; L. von Smekal, A. Hauck and R. Alkofer, Ann. Phys. 267 (1998) 1; L. vonSmekal, A. Hauck and R. Alkofer, Phys. Rev. Lett. 79 (1997) 3591.

5. A. C. Aguilar and A. A. Natale, hep-ph/0405024; JHEP 0408 (2004) 57. 
Copyright of AIP Conference Proceedings is the property of American Institute of Physics and its content may not be copied or emailed to multiple sites or posted to a listserv without the copyright holder's express written permission. However, users may print, download, or email articles for individual use. 